\title{
Diagnóstico energético de la empresa TEIMSA basado en la norma ISO 50001
}

\author{
Gloria Elizabeth Miño Cascante ${ }^{1}$ \\ Ángel Geovanny GuAmán Lozano ${ }^{2}$ \\ Julio CÉsar Moyano Alulema ${ }^{3}$ \\ TATIANA JaCKeline ORTIZ GonZÁLEZ ${ }^{4}$ \\ Eduardo Francisco García Cabezas ${ }^{5}$
}

\begin{abstract}
RESUMEN
En esta investigación se formulan los estándares de producción en correlación directa con el consumo energético, en este proceso se utilizan las herramientas proporcionadas por la norma ISO 50001 orientadas a cumplir con las iniciativas de mejora del rendimiento energético, optimización del sistema de gestión de la información de energía y auditoría dentro de la empresa Textiles Industriales Ambateños S. A TEIMSA. Mediante el monitoreo de los consumos energéticos se determina la eficiencia de máquinas, la toma de acciones referentes a cambios, mejoras y proyectos que mejoren las métricas de la empresa. Como resultado se llega a construir las líneas base de consumo y se plantean metas que nos permitan manejar una eficiente gestión de recursos energéticos mediante la aplicación de indicadores mensurables de energía y validada mediante un juicio de expertos. Finalmente se concluye que la aplicación de la primera fase de la norma refleja los principales sujetos de consumo energético dentro de la empresa y traza la ruta para el mejoramiento continuo en la gestión de la empresa.
\end{abstract}

Palabras-claves: Líneas base de consumo; indicadores energéticos; ISO 50001.

ENERGY DIAGNOSIS OF THE COMPANY TEIMSA BASED ON ISO 50001

\section{ABSTRACT}

In this research the production standards are formulated in direct correlation with the energy consumption, in this process the tools provided by the standard ISO 50001 are used to comply with the initiatives of improvement of the energy efficiency, optimization of the system of information management energy and auditing within the company "Textiles Industriales Ambateños" $\mathrm{S}$. A. TEIMSA. By monitoring energy consumption, the efficiency of machines is determined, actions are taken regarding changes, improvements and projects that improve the company's metrics. As a result, we get to build the consumption baselines and set goals that allow us to manage an efficient management of energy resources through the application of measurable indicators of energy and validated by an expert judgment. Finally, it is concluded that the application of the first phase of the standard reflects the main subjects of energy consumption within the company and outlines the route for continuous improvement in the energy management of the company.

Keywords: Baseline consumption; energy indicators: ISO 50001.

\section{INTRODUCCIÓN}

La situación actual de alta competitividad y globalización de los mercados exige a las empresas cambiar sus estrategias para adaptarse a las nuevas tendencias. Los requerimientos del cliente, la aceleración del cambio tecnológico y los ciclos de vida de los productos cada vez más cortos son elementos de entorno que obligan a las organizaciones a mejorar la variedad, calidad, plazos y costos de sus (Vásquez et al, 2017) productos o servicios. Una de las estrategias para optimizar los recursos es la eficiencia energética que tiene como propósito promover la reducción del consumo global de los consumidores de energía (Correa et al, 2014), alentando a los consumidores de energía a adoptar equipos o sistemas con alta eficiencia y proporcionando el mismo efecto en comparación con los equipos anteriores. (Lee, Hyeong, Jong, Jae y Myeong, 2013).

De forma paralela múltiples estudios revelan los temores ambientales (Dzene, Polikarpova, Zogla y Marika, 2015) y la preocupación por el ecosistema mundial que pueden aprovecharse para fomentar la eficiencia energética. (Malpas, 2011) (Tallini y Cedola, 2016) Manifiesta que, aunque todavía hay mucha incertidumbre en torno a las causas y las soluciones al efecto invernadero, las emisiones de dióxido de carbono de $\mathrm{CO} 2$ son claramente una fuente importante de preocupación. Por estas razones, las emisiones de carbono son un componente crucial de la eficiencia energética (Katchasuwanmanee, Bateman y Cheng, 2017).

Las limitaciones causadas por el consumo de energía cada vez mayor y la variación del precio internacional del petróleo en el campo de la economía se han convertido en problemas mundiales según manifiesta Lee , Hyeong, Jong, Jae y Myeong (2013) y afectan a la gestión energética de las organizaciones (Larios, 2014). Incluso algunas de las industrias como las producciones

Magister en Dirección de Empresas mención proyectos, Doctor en Ciencias Económicas, Vicerrectora Académica de la Escuela Superior Politécnica de Chimborazo. Docente Investigador de la Facultad de Ingeniería Industrial de la Escuela Superior Politécnica de Chimborazo, Riobamba-Ecuador. E-mail: gmino@espoch.edu.ec

2 Magister en Gestión Industrial y Sistemas Productivos. Docente investigador de la Carrera de Ingeniería Industrial - Facultad de Mecánica de la Escuela Superior Politécnica de Chimborazo. Riobamba-Ecuador. E-mail a guaman@espoch.edu.ec. ORCID iD: https://orcid.org/0000-0002-5145-6994

3 Magister en Gestión Industrial y Sistemas Productivos, Magister en Seguridad Industrial. Docente investigador de la Carrera de Ingeniería Industrial - Facultad de Mecánica de la Escuela Superior Politécnica de Chimborazo. Riobamba-Ecuador. E-mail j_moyano@espoch.edu.ec. ORCID iD: https://orcid.org/0000-0001-6672-9409

4 Investigadora de la Carrera de Ingeniería Industrial - Facultad de Mecánica de la Escuela Superior Politécnica de Chimborazo. Riobamba-Ecuador. E-mail t_ortiz@espoch.edu.ec

5 Magister en Sistemas de Control y Automatización Industrial. Docente investigador de la Carrera de Ingeniería Industrial - Facultad de Mecánica de la Escuela Superior Politécnica de Chimborazo. Riobamba-Ecuador. E-mail egarcia@espoch.edu.ec. ORCID iD: https://orcid.org/0000-0002-3547-472X 
avícolas han implantado de forma exitosa sistemas con el ánimo de utilizarlos en un proceso de gestión de la producción y proporcionar energía limpia (Bornschlegla, Bregulla y Frankeb, 2016) para satisfacer una parte de la demanda de energía según manifiesta (Killic, 2016).

Hay un mito generalizado entre las personas de que la adaptación de un sistema de gestión de la energía sería una carga adicional, pero a diferencia de todos los mitos, es realmente fácil mantener un sistema de gestión de la energía (Sundararaj,Karthick y Ponrajkumar, 2016).

En muchos casos el ahorro potencial de energía (McKane, Therkelsen, Scodel, Prakash, Aghajanzadeh y O'Sullivan, 2017) es grande, pero las barreras a superar también son altas es por eso que muchos propietarios consideran que los beneficios de las medidas no superan los costos (Visscher,Meijer y Maltard, 2016) y la inversión establecida.

En el corto y mediano plazo, también es necesario considerar el desarrollo de tecnologías avanzadas de generación de energía. Para ello es aconsejable llevar a cabo estudios de viabilidad para soluciones ( Lisin, Strielkowski y Garanin, 2015) además del trabajo que debe hacerse con los consumidores intermedios y finales para diseñar las políticas adecuadas para explotar las oportunidades y debe estar respaldado por la incorporación del conocimiento de los flujos de emisiones incorporados a la política, así como a la investigación.

Es necesario seguir estudiando el impacto de esta nueva perspectiva en la formulación de políticas ( Beihmanis y Marika, 2016), debe prestarse especial atención a la aplicación práctica de las políticas que abordan las emisiones incorporadas, incluidos los procedimientos contables y los requisitos administrativos para medir y supervisar las emisiones de la cadena de suministro que atraviesan las fronteras internacionales (Scott, Roelich, Owen y Barre$\mathrm{tt}, 2017$ ). Los mecanismos exactos para asegurar cualquier superposición entre las políticas son complementarios para lograr una reducción absoluta de las emisiones y no debilitan las políticas existentes. (Acosta, González , Zamarreño y Álvarez, 2011)

La identificación, entendimiento y administración de los procesos interrelacionados como un sistema contribuye a la eficacia y eficiencia de las organizaciones. Un método eficiente para que las empresas organicen sus actividades orientadas a cumplir con la misión de ahorro energético es la Norma ISO 50001 (Dörr, Wahren y Bauernhansl, 2013), (Hang, 2015) ésta tiene el propósito de "permitir a las orga- nizaciones establecer los sistemas y procesos necesarios para mejorar el rendimiento energético, incluida la eficiencia energética, el uso y el consumo" (Johnson, Johansson, Andersson y Södahl, 2013).

La presente investigación se orienta a implementar el sistema ISO 50001 en la empresa TEIMSA dedicada a la fabricación, tinturado, acabados y comercialización de hilos "open end" y tela de algodón al $100 \%$. Esta empresa manufacturera tiene la necesidad de satisfacer las exigencias del mercado desarrollando un modelo de gestión energética basado en control de procesos como un elemento estratégico para mejorar la eficiencia y calidad en todas sus áreas. La documentación es el soporte del sistema de gestión energética, en ella no solo se plasman las formas de operar de la empresa (Therkelsena, Masanetb y Worrellc, 2014), sino toda la información sobre los elementos básicos, responsabilidades, maquinaria y métodos de control, y la toma de decisiones. Un sistema de gestión de la energética debe ser considerado como un plan diario aplicable en la empresa (Schudeleit, Züst, Weiss y Wegener, 2016) para conseguir las metas establecidas, ésta depende de la participación de todos los miembros de la empresa.

\section{METODOLOGÍA}

La metodología de la investigación utiliza esquemas tradicionales de gestión de energía en combinación con las disposiciones de la ISO 50001. El modelo conceptual adaptado de ISO Sistemas de Gestión de Energía describe varias fases que se muestran en la Figura 1.

Las fases que se sigue la metodología detallada está conformada por una política energética, planificación de la energía, auditoría, aplicación y funcionamiento y son seguidos por una fase de control constante. Esto a su vez es apoyado por un sistema de auditoría interna, procesos de monitoreo, medición y análisis, así como para las no conformidades, la corrección, la acción correctiva y preventiva.

\section{Criterios para la revisión energética}

La norma ISO 50001, indica que se deben investigar los siguientes criterios como parte de la revisión energética en una empresa:

1. Análisis de Consumo y Uso de la Energía

2. Identificación de Usos Significativos de Energía (USEn)

3. Análisis de Oportunidades de Desempeño Energético (EPOD) 


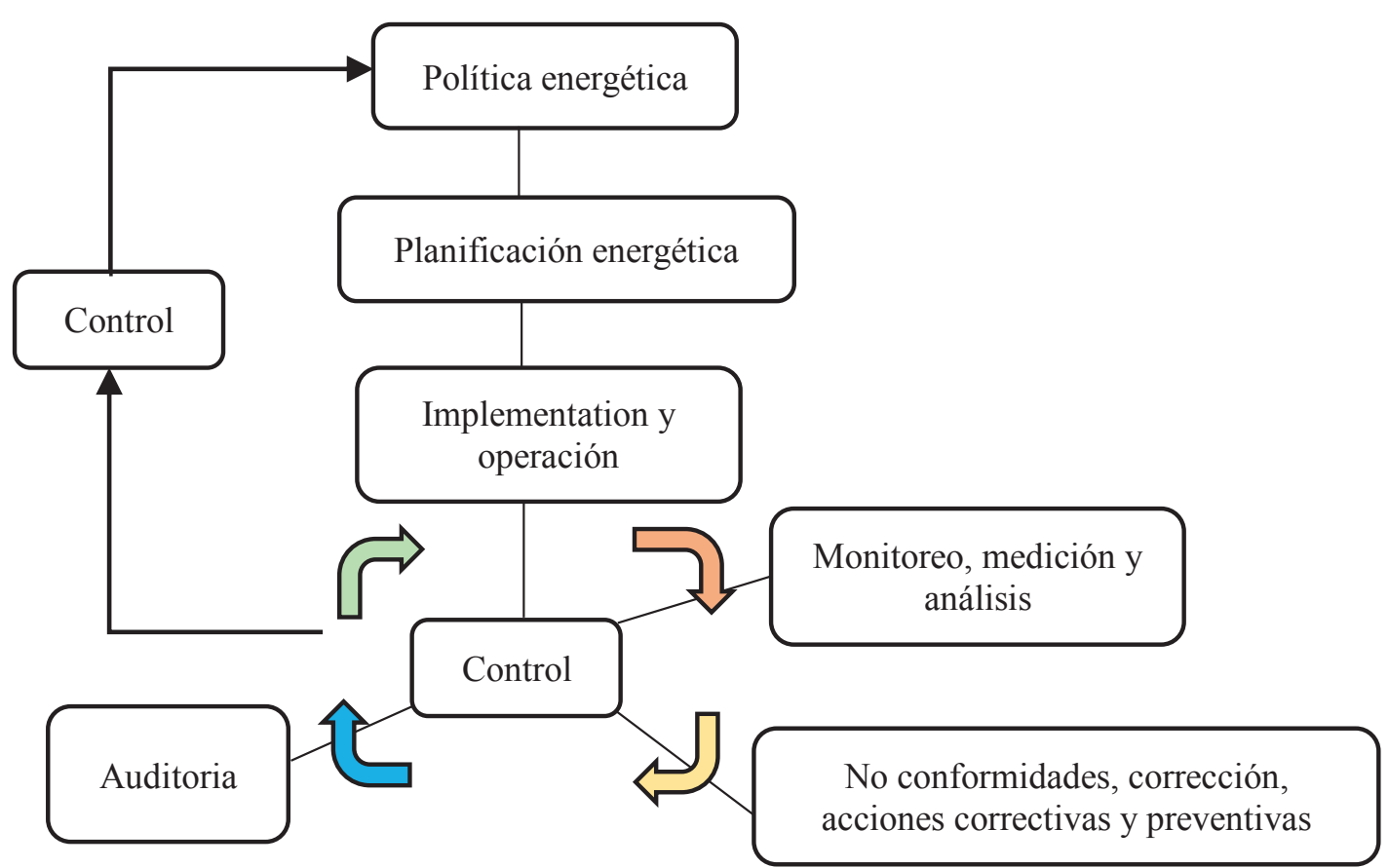

Figura 1. Metodología del sistema de gestión energética.

Fuente: Elaboración propia.

\section{Análisis de consumo y uso de la energía}

Es la identificación y el análisis de los datos existentes basados en mediciones existentes de los diferentes tipos de energía identificadas en la empresa, para este caso en particular la referencia son las facturas canceladas a los proveedores. Las fuentes energéticas primarias (fuentes de energía útiles) son las que se obtienen a partir de un proceso de transformación físico o químico de una fuente primaria de energía. En base a la recopilación de información del departamento financiero de la empresa las fuentes energéticas primarias son: la energía eléctrica, agua, diesel y GLP ilustrado en la tabla1.

\section{Recolección de la información}

La información para realizar el diagnóstico energético de la empresa está basada en la siguiente matriz de documentación véase tabla 1 :

Tabla 1. Principales fuentes de información para el diagnóstico

\begin{tabular}{|c|c|c|}
\hline PROCESO & $\begin{array}{c}\text { DOCUMENTO REQUERIDO PARA EL } \\
\text { ANÁLISIS }\end{array}$ & DOCUMENTO GENERADO PARA EL DIAGNÓSTICO \\
\hline \multirow{5}{*}{$\begin{array}{l}\text { ANÁLISIS DE FACTURAS } \\
\text { DE ENERGÍA ELÉCTRICA }\end{array}$} & FACTURAS DE ENERGÍA ELÉCTRICA & $\begin{array}{l}\text { Base de datos de control mensual de valores de la planilla de } \\
\text { energía eléctrica }\end{array}$ \\
\hline & \multirow{2}{*}{ EASA } & Análisis de factor de potencia (FP) \\
\hline & & Análisis de factor de corrección (FC) \\
\hline & \multirow{2}{*}{ SAP } & Análisis de penalización por FP y FC, acumulación mensual \\
\hline & & Tendencia anualizada de consumo de energía activa \\
\hline \multirow{3}{*}{$\begin{array}{l}\text { ANÁLISIS DE FACTURAS } \\
\text { DE DIÉSEL }\end{array}$} & \multirow{3}{*}{$\begin{array}{l}\text { Facturas mensuales de proveedor } \\
\text { Consumos en SAP }\end{array}$} & Base de datos de consumo mensual de diésel \\
\hline & & Base de datos de consumo diario de diésel en los calderos \\
\hline & & Gráfica de consumo diario de diésel y agua en los calderos \\
\hline
\end{tabular}

Fuente: Elaboración propia. 


\section{Análisis del consumo de energía}

El análisis inicial de costos de consumos energéticos es realizado en base a la información suministrada por las facturas de cada uno de los proveedores de las fuentes de energía, los mismos que están concentrados en el área financiera de tal forma que para la empresa la energía representa un gasto, y no un costo.

\section{RESULTADOS Y DISCUSIÓN}

Con los datos suministrados por el área financiera ilustrada en la figura 2 es posible determinar los porcentajes de costos anuales que la empresa gasta en energéticos requeridos para sus operaciones.
El principal consumo de energía está concentrado en la energía eléctrica, de tal forma que el Equipo de Gestión de la Energía, enfoca el análisis del consumo eléctrico en la planta. Las tarifas de consumo eléctrico para los clientes residenciales, industriales, entre otros; están determinados en el pliego tarifario conforme a las disposiciones de la Ley de Régimen del Sector Eléctrico, el Reglamento General de la Ley, la Codificación del Reglamento de Tarifas Eléctricas y la Ley Orgánica de la Ley del Consumidor y su Reglamento en la República del Ecuador.

La figura 3 muestra la tendencia de consumo de la energía eléctrica de acuerdo a demanda horaria de acuerdo a los rangos tarifarios establecidos en el pliego tarifario.

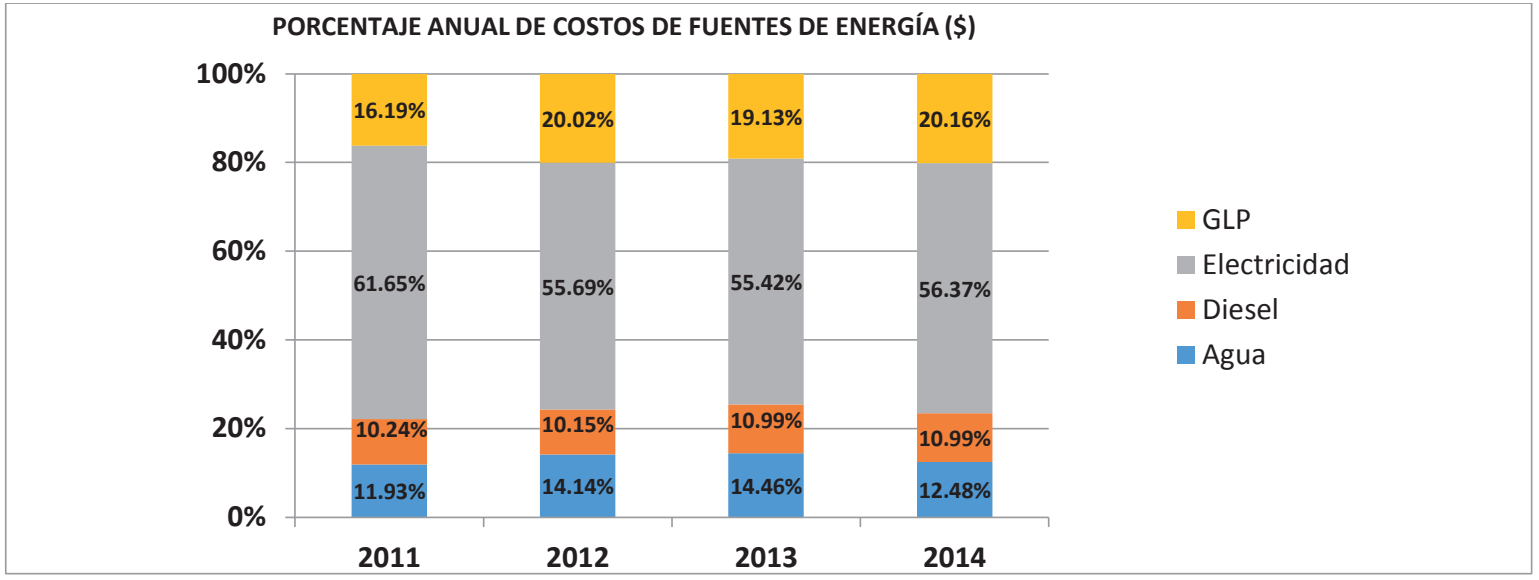

Figura 2. Distribución de costos anuales de las fuentes de energía.

Fuente: Elaboración propia información del área financiera

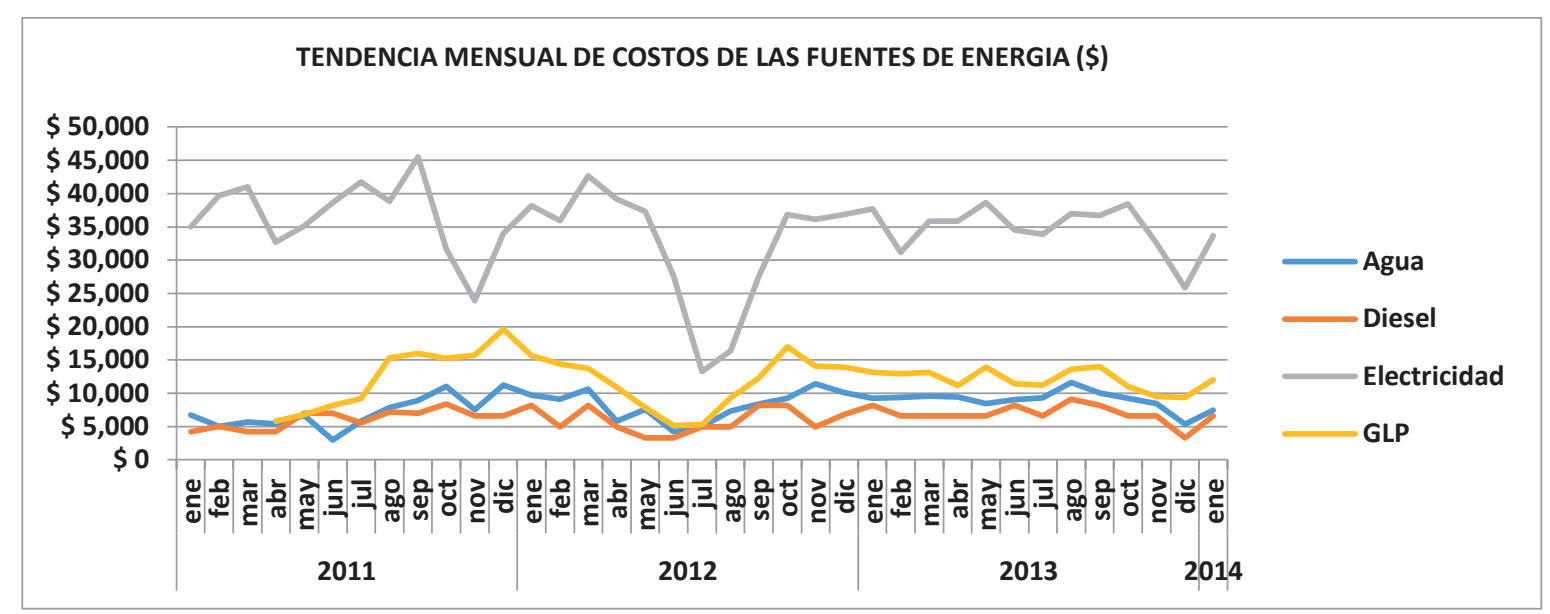

Figura 3. Variación de los costos mensuales de la energía.

Fuente: Elaboración propia. 
El Consejo Nacional de Electricidad aplica una tarifa diferenciada a los consumidores industriales que tienen un registrador de demanda horaria en donde son identificables los consumos de potencia y energía en los períodos horarios de punta, media y base con el propósito de incentivar los consumos de energía en las horas de menor demanda de 22:00 a 08:00, en la figura 4 ilustra el consumo de energía por bandas horarias.

\section{Cargo por bajo factor de potencia y factor de corrección.}

Los consumidores industriales que tienen medición con registrador de demanda, con medición de energía reactiva, que registren un factor de potencia media mensual inferior a 0.92, deben ser penalizados. En la empresa no se presenta esta situación porque el FP en el medidor principal está en promedio de 0.94. El pliego tarifario también incluye al Factor de Corrección (FC), y la define como la relación entre la demanda en horario pico y la demanda máxima registrada en el mes en el medidor registrador. La organización tiene una tendencia a ser penalizada con un FC de 1.2 al valor de la demanda máxima registrada, como se indica en la figura 5 en el que se analiza el factor de potencia que tiene la empresa.

En la actualidad TEIMSA, está cancelando un valor adicional del $20 \%$ por cada $\mathrm{kW}$ de potencia registrada, es decir, que cuando el costo de kW de potencia máxima registrada es de USD. $4^{54 / 100 / ~ k W ; ~}$ la empresa cancela el valor de USD. $5^{45 / 100}$, desde el año 2011 hasta noviembre de 2013, la empresa estima una penalización de aproximadamente de

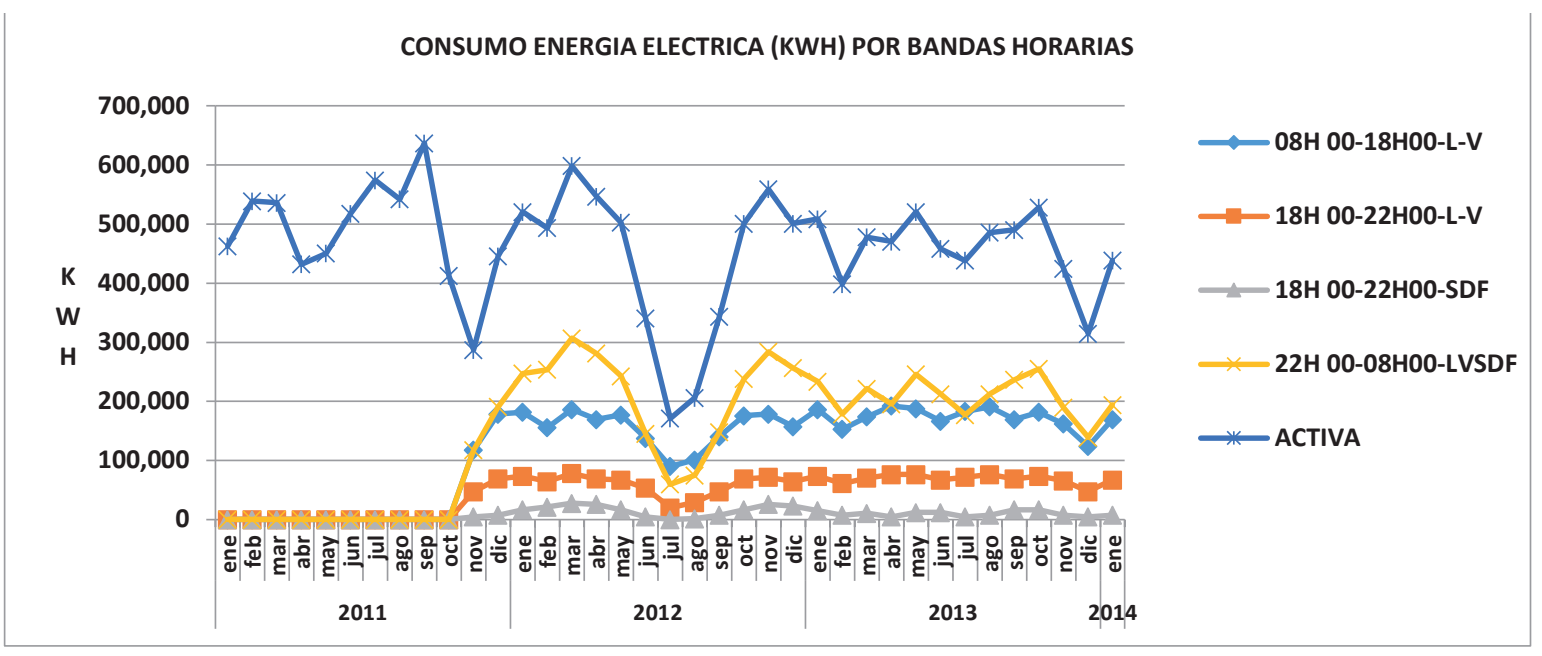

Figura 4. Variación del consumo de energía eléctrica en cuatro periodos Fuente: Elaboración propia.

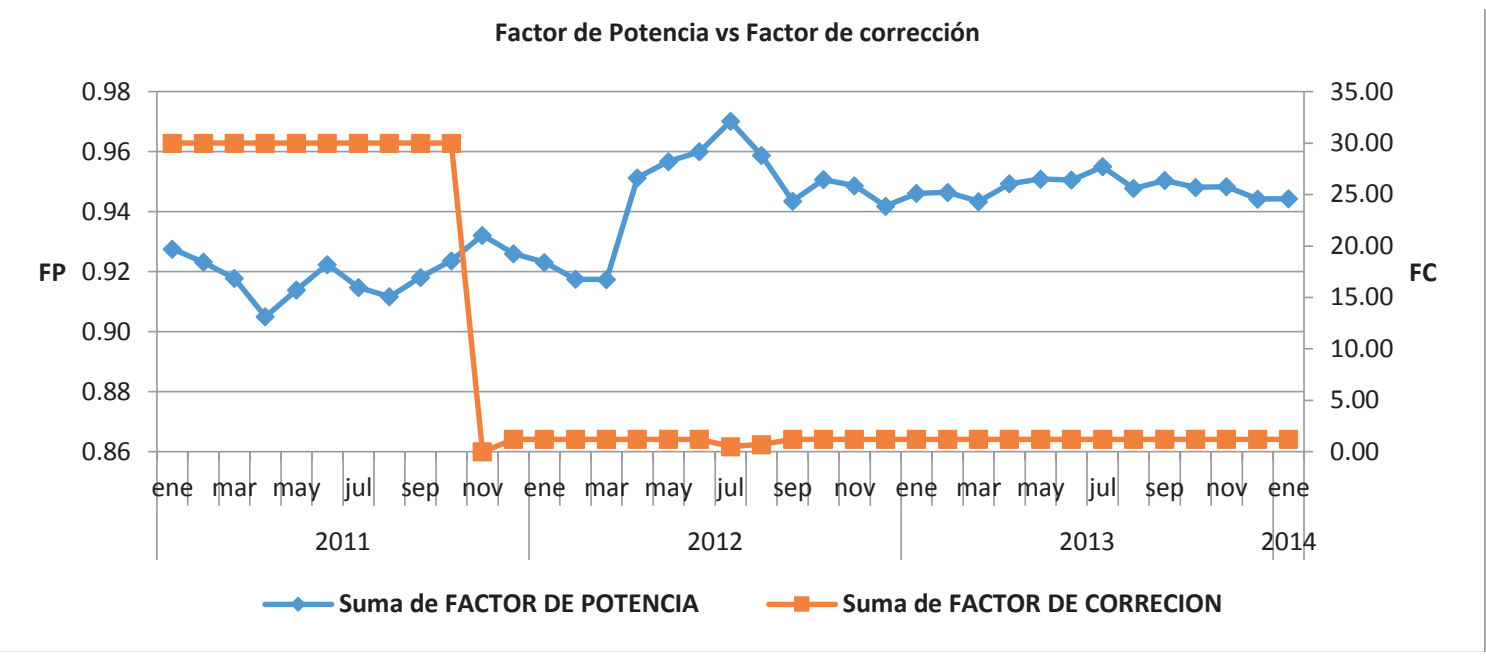

Figura 5. Factor de potencia y corrección por periodos

Fuente: Elaboración propia. 
USD. 50000,00. Otra fuente de energía principal en la planta es el diésel, este combustible es consumido por los dos calderos disponibles para generar vapor (Caldero Kewanee 200 BHP y Caldero Gavardo $200 \mathrm{BHP}$ ), la siguiente gráfica indica la tendencia de comportamiento del consumo de diésel en la empresa. Existe disminución del consumo de diésel entre mayo y septiembre de 2012, la causa para el bajo consumo fue el mantenimiento correctivo programado del caldero Kewanee de $200 \mathrm{BHP}$, el tiempo de mantenimiento de la caldera fue de aproximadamente tres meses, y como se aprecia en la gráfica la caldera entró en operación otra vez en octubre de 2012 en consecuencia el consumo de diésel volvió a niveles normales. Los datos de producción de los meses son altos respecto al resto del año en mención, además se identifican algunos problemas cuando trabaja el caldero Gavardo (Acuatubular): a. El personal de producción evidencia que existe arrastre en la tubería de vapor.

b. Problemas en la calidad del producto elaborado en la sección.

Otro tipo de combustible fósil consumido en las operaciones en la planta industrial es el Gas Licuado de Petróleo (GLP), es muy importante analizar la tendencia del consumo del GLP debido a que un solo usuario energético, en la figura 6 se ilustra el consumo mensual de la empresa.

La figura 7 muestra el consumo energético en BTU en la planta industrial de TEIMSA obtenida mediante el análisis de la información de las facturas de las fuentes de energía primarias en unidades de energía similares, por lo cual se transformó las unidades de venta de los proveedores en unidades de energía de uso en la industria para determinar la tendencia de consumo energético en la planta industrial.

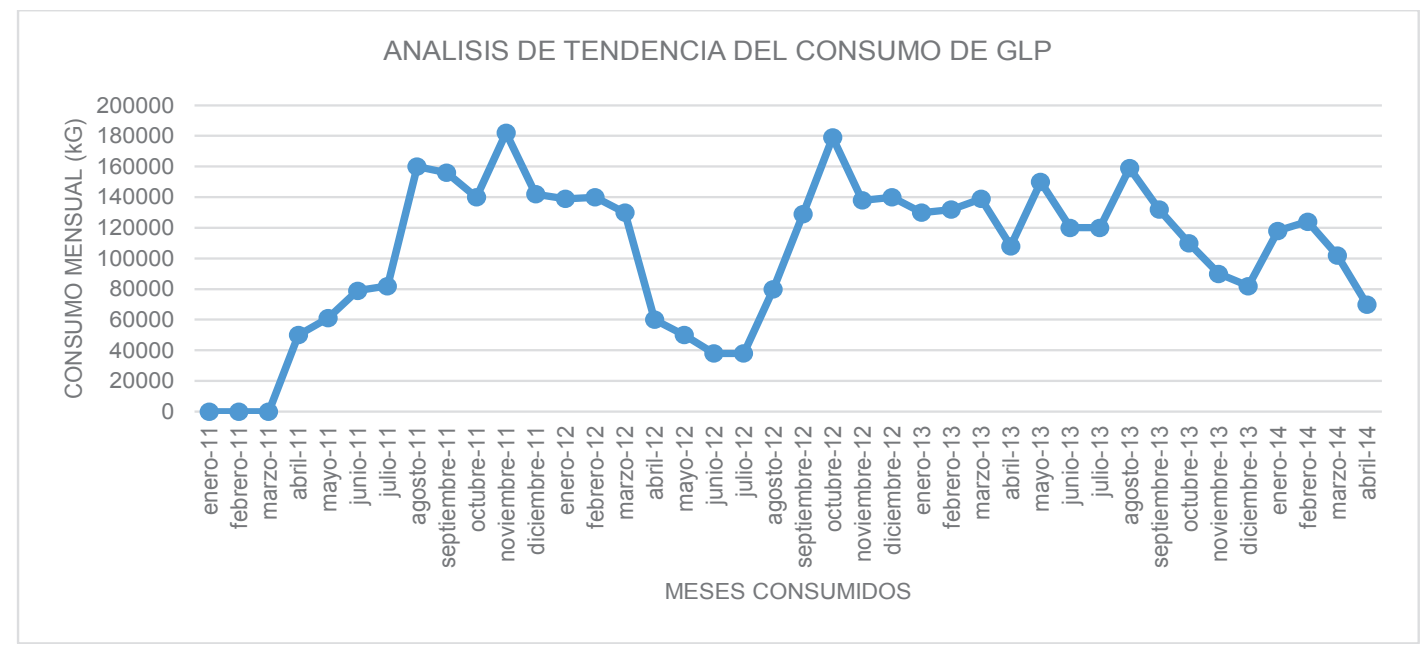

Figura 6. Evolución en el consumo de GLP. Fuente: Elaboración propia.

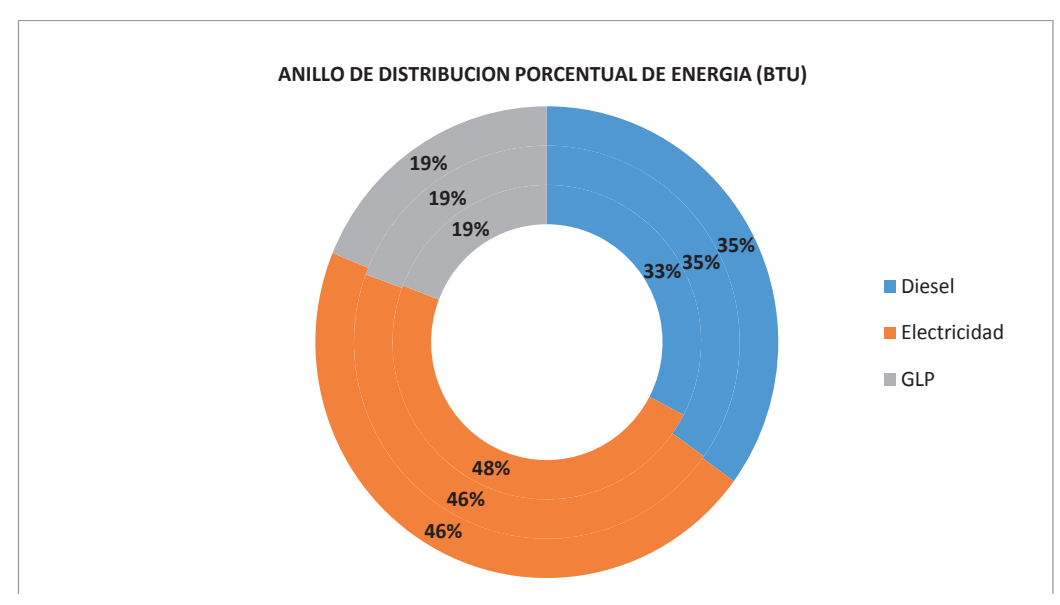

Figura 7. Distribución porcentual de energía en BTU’s

Fuente: Elaboración propia. 


\section{Variables que afectan a los usuarios significati-} vos de energía.

Para identificar las variables que están afectando a los usuarios significativos de energía se puede observar en la siguiente tabla 2 donde se categoriza los criterios de priorización y valoración, teniendo en cuenta el consumo energético, el costo energético, el factor de carga y el factor de servicio:

Luego de evaluar la matriz de los usuarios energéticos con la aplicación los criterios se obtiene la lista usuarios en la planta. El análisis de los usuarios energéticos es realizado hasta el nivel de áreas de producción, por no disponer de elementos de medición en cada una de las máquinas, los Usuarios Significativos de Energía son los siguientes: planta de Hilatura, Tisaje y de Acabados. La base de datos de información a nivel de máquinas permite obtener información a mayor detalle siendo posible identificar los principales usuarios de energía en cada una de las áreas de producción.

\section{Presupuesto de energía utilizando líneas base energéticas}

El cálculo para el presupuesto de energía se realiza utilizando la línea base energética de cada una de las fuentes de energía, considerando el valor de producción como la variable dependiente en el cálculo de la ecuación y respetando las siguientes consideraciones:

a. Línea base. - Es la base para evaluar las mejoras en el desempeño energético, y es aplicable a toda la instalación, sistemas, áreas, equipos, USEn. b. Indicadores de desempeño energético. - Son los que se usan para medir el desempeño energético. Ejemplos: IDEn para toda la empresa, por áreas / procesos, por sistema energético.

c. Producción equivalente. - Es determinar el número de $\mathrm{kg}$ de producción., elaborados en función del porcentaje de energía usada en el área de procesamiento. Es posible utilizar la siguiente ecuación:

$$
P E=\sum_{1}^{n} \frac{\text { Energía }_{U S E n_{n}} * K g_{U S E n_{n}}}{\% \text { Energía Total }}
$$

Donde:

PE: Producción equivalente

\%Energía usEn $n$ : porcentaje de energía usada por el USEn

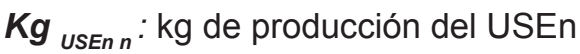

\%Energía Total usEn : suma de los porcentajes de todos los USEn considerados

De acuerdo al análisis en la figura 8 se determina la relación que debe existir entre el consumo de la planta en BTUs que identifica el consumo actual de la planta de textiles estableciendose una relación lineal. Para la determinación se la línea meta de consumo de diésel se utilizan coeficientes de corrección y se limitan los rangos de consumo y sirvan de indicadores para el diagnóstico de la situación de la planta.

Tabla 2. Criterios de valoración para usuarios energéticos

\begin{tabular}{|l|l|l|l|}
\hline \multicolumn{3}{|c|}{ PRIORIZACIÓN USUARIOS ENERGÉTICOS } \\
\hline \multirow{2}{*}{ CRITERIOS } & DE PRIORIZACIÓN Y VALORACIÓN PARA LOS USUARIOS ENERGÉTICOS \\
\cline { 2 - 4 } & IMPACTO ALTO & IMPACTO MEDIO & IMPACTO BAJO \\
\cline { 2 - 4 } & 5 puntos & 3 puntos & 1 punto \\
\hline Consumo energético & Mayor a $5 \%$ & Entre $2 \%$ y $5 \%$ & Menor al $2 \%$ \\
\hline Costo energético & Mayor a $\$ 1,00$ & Entre $\$ 0.50$ y $\$ 1.00$ & Menor a $\$ 0.50$ \\
\hline Factor de carga & Mayor al $85 \%$ & Entre el $60 \%$ y $85 \%$ & Menor al $60 \%$ \\
\hline Factor de servicio & Tres turnos & Dos turnos & 1 turno \\
\hline
\end{tabular}

Fuente: Elaboración propia. 


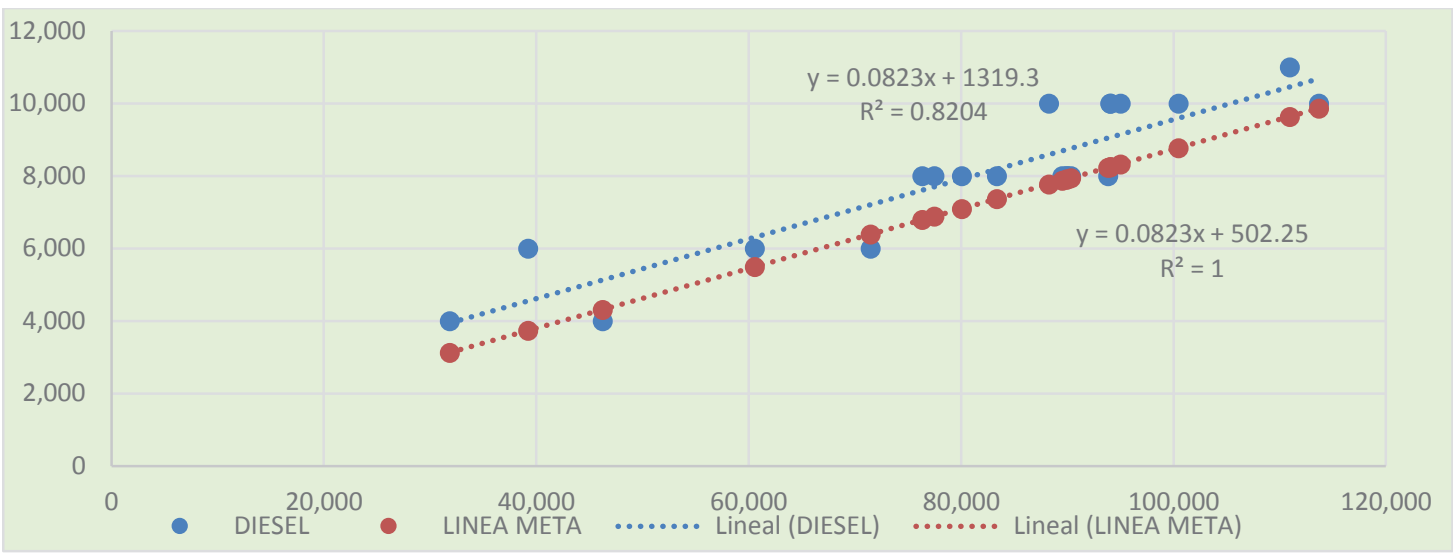

Figura 8. Determinación de la línea meta de consumo de diésel

Fuente: Elaboración propia.

Posteriormente se establecen los nuevos coeficientes descritas en la tabla 3 para la determinacion de la linea meta del consumo de diesel, cuyos valores serviran para determinar una línea base en el inicio de una adecuada gestíon energética.

Como fruto final de la investigación se establecen las ecuaciones de la tabla 4 que servirán para que la empresa identifique vulnerabilidades en el consumo energético de la planta de textiles. Este proceso debe periódicamente ser calibrado por los analistas.

La fase final del estudio fue la incorporación de un juicio de expertos como se muestra en la tabla 5 , que sirvió para validar la metodología y su la aplicabilidad en función de siete variables como son: Claridad, objetividad, consistencia, coherencia, pertinencia, suficiencia y relevancia.

El cuestionario tiene un grado de confiabilidad de 0.8106 correspondiente al análisis con el alfa de Cronbach. En general, los resultados reflejan que los ocho expertos califican a la metodología en las escalas "Buena" y "Muy buena" como se ve en la figura 9, lo que hace que el instrumento pueda ser usado con fidelidad para realizar la identificación de los principales agentes energéticos de una organización.

Tabla 3. Coeficientes para la determinación de la línea base de consumo

\begin{tabular}{|l|c|}
\hline DESCRIPCIÓN & VALOR \\
\hline B=m & 0,0823 \\
\hline A & 1319,3 \\
\hline N & 11 \\
\hline$A_{\circ}$ & 817,05 \\
\hline$E_{o}$ & 502,25 \\
\hline Promedio consumo real & 7.731 \\
\hline Potencial real & $10,57 \%$ \\
\hline
\end{tabular}

Fuente: Elaboración propia.

Tabla 4. Fórmulas para el monitoreo de Usuarios Energéticos

\begin{tabular}{|c|c|c|c|c|}
\hline IDEn & Sección & Energía Teórica LíNEA BASE & Energía Real & I BASE 100 \\
\hline $\mathrm{MBTU} / \mathrm{kg}$ & Calderas & $\varepsilon L B=0,0823^{*} \mathrm{~kg}_{0}+1292,1$ & $\varepsilon r=$ Consumo de Combustible según Factura & $\varepsilon \mathrm{LB} / \varepsilon r * 100$ \\
\hline $\mathrm{MBTU} / \mathrm{kg}$ & Rama & $\varepsilon L B=0,1321^{*} \mathrm{~kg}_{0}+2444,1$ & $\varepsilon r=$ Consumo de GLP según Factura & $\varepsilon \mathrm{LB} / \varepsilon r * 100$ \\
\hline $\mathrm{kWh} / \mathrm{kg}$ & Open End & $\varepsilon \mathrm{LB}=0,706^{*} \mathrm{~kg}_{0}+11133$ & $\varepsilon r=$ Consumo de Energia*38,72\% & $\varepsilon \mathrm{LB} / \varepsilon r^{*} 100$ \\
\hline
\end{tabular}

Fuente: Elaboración propia. 
Tabla 5. Cuestionario de validación

\begin{tabular}{|c|c|c|c|c|c|c|c|}
\hline & & \multicolumn{5}{|c|}{ VALORACIÓN } \\
\hline & & & Muy malo & Malo & Regular & Bueno & Muy Bueno \\
\hline & & CRITERIO & 1 & 2 & 3 & 4 & 5 \\
\hline \multirow{7}{*}{1} & Claridad & $\begin{array}{l}\text { El instrumento cuenta con un lenguaje apropiado que } \\
\text { facilita su comprensión }\end{array}$ & & & & & 1 \\
\hline & Objetividad & La metodología tiene conductas observables, medibles & & & & 1 & \\
\hline & Consistencia & $\begin{array}{l}\text { La información se organización de forma lógica y en } \\
\text { base a los fundamentos teóricos de eficiencia energética }\end{array}$ & & & & 1 & \\
\hline & Coherencia & $\begin{array}{l}\text { Existe relación de los contenidos con los indicadores } \\
\text { de la variable }\end{array}$ & & & & 1 & \\
\hline & Pertinencia & La escala de los indicadores son apropiados & & & & & 1 \\
\hline & Suficiencia & $\begin{array}{l}\text { Son suficientes la cantidad y calidad de variables utili- } \\
\text { zadas en la metodología }\end{array}$ & & & 1 & & \\
\hline & Relevancia & $\begin{array}{l}\text { El método brinda un aporte en la medición de la eficien- } \\
\text { cia energética de una empresa }\end{array}$ & & & & 1 & \\
\hline & & SUMA PARCIAL & 0 & 0 & 3 & 16 & 10 \\
\hline & & SUMA TOTAL & 29 & & & & \\
\hline
\end{tabular}

Fuente: Elaboración propia.

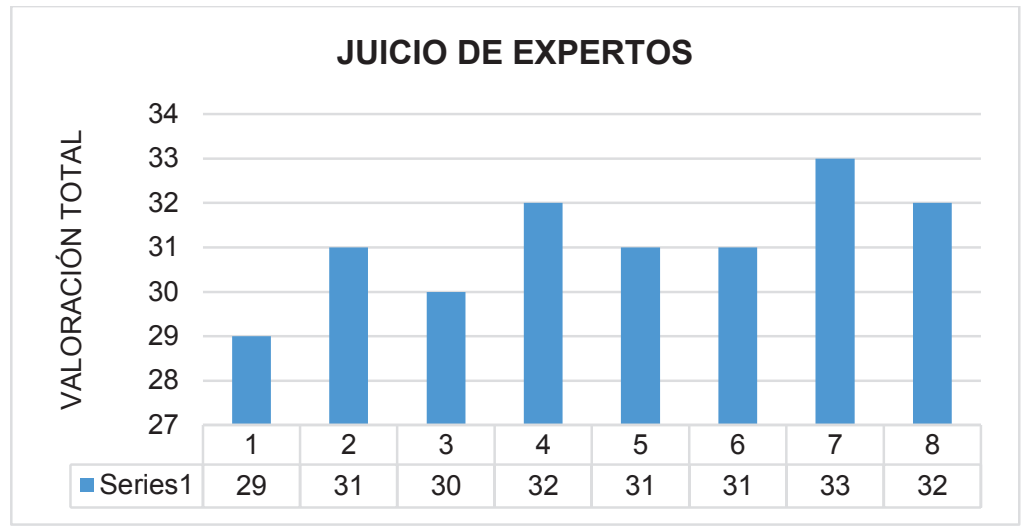

Figura 9. Resultados totales del análisis de expertos

Fuente: Elaboración propia.

\section{CONCLUSIONES}

Los procedimientos la norma internacional ISO 50001 son flexibles y pueden ser aplicados con éxito a organizaciones industriales para el diagnóstico energético de las empresas. Por otra parte, la disponibilidad de datos es crucial para planificar las acciones de mejora. La falta de datos puede resolverse mediante la implementación de procedimientos integrados de gestión de datos como parte de un sistema de gestión de energía, además dicha información debe utilizarse para calcular indicadores de energía mensurables. En el análisis eléctrico evidencia que la demanda de energía en las horas pico no tiene control, de tal forma que la empresa tiene un potencial de ahorro de un $36 \%$ del costo de la demanda facturable mensual, mediante la aplicación de políticas de eficiencia energética.

\section{REFERENCIAS BIBLIOGRÁFICAS}

Acosta, A., González, A., Zamarreño, J. y Álvarez V. (2011). Modelo para la Predicción Energética de una Instalación Hotelera. Revista Iberoamericana de Automática e Informática Industrial RIAI ,8(4),309-322.doi: 10.1016/j. riai.2011.09.001.

Bornschlegla, M., Bregulla, M. y Frankeb, J. (2016). Methods-Energy Measurement - An approach for sustainable energy planning of manufacturing technologies. Journal of Cleaner Production, 135(1), 644-656. doi: 10.1016/j. jclepro.2016.06.059.

Beihmanis, K. y Marika R. (2016). Energy Management System Implementation in Latvian Municipalities: From Theory to Practice. 
Energy Procedia. 95(1), 66-70. doi: 10.1016/j. egypro.2016.09.018

Correa, J., Borroto, E., Alpha, M., González, R., Curbelo, M. y Díaz A. (2014). Diseño y aplicación de un procedimiento para la planificación energética según la NC-ISO 50001:2011, Ingeniería Energética, 35(1), 38-47. Recuperado en 04 de enero de 2018, de http://scielo.sld.cu/scielo.php?script=sci arttext\&pid=S1815-59012014000100005\&Ing= es\&tlng=es.

Dzene, I., Polikarpova, I., Zogla, L. y Marika, R. (2015). Application of ISO 50001 for implementation of sustainable energy. Energy Procedia, 72, 111-118. doi: 10.1016/j. egypro.2015.06.016

Dörr, M.,Wahren, S. y Bauernhansl, T. (2013). Methodology for Energy Efficiency on Process Level, Procedia CIRP, 7, Pages 652-657. doi: 10.1016/j.procir.2013.06.048

Hang, H. (2015). Energy management systems and market value: Is there a link?, Economic Modelling, 46, 70-78. doi:10.1016/j. econmod.2014.12.038

Johnson, H., Johansson, M., Andersson, K. y Sodahl, B. (2013). Will the ship energy efficiency management plan reduce $\mathrm{CO} 2$ emissions? A comparison with ISO 50001 and the ISM code. Maritime Policy \& Management, 40(2), 177190. doi: 10.1080/03088839.2012.757373

Katchasuwanmanee, K., Bateman, R. y Cheng, K. (2017). An Integrated approach to energy efficiency in automotive manufacturing systems: quantitative analysis and optimization. Production \& Manufacturing Research, 5(1) 9098. doi:10.1080/21693277.2017.1322539.

Killic, I. (2016). Analysis of the energy efficiency of poultry houses in the Bursa region of Turkey. Journal of Applied Animal Research, 44(1), 165172. doi: 10.1080/09712119.2015.1021813.

Larios. A. (2014). La energía renovable en México: perspectivas desde el Balance Nacional de Energía 2012, Economía Informa, 385, 90-99, doi: 10.1016/S0185-0849(14)70423-2.

Lee, W., Hyeong, K., Jong, P., Jae, P. y Myeong, C. (2013). Development of Investment Strategies of Energy Efficiency Programs in Korea. Journal of International Council on Electrical Engineering, 3(1), 38-44. doi: 10.5370/ JICEE.2013.3.1.038.
Lisin, E., Strielkowski, W. y Garanin, I. (2015). Economic efficiency and transformation of the Russian energy sector. Economic ResearchEkonomska Istraživanja. 28(1), 620-630. doi: 10.1080/1331677X.2015.1086886.

Malpas, R. (1989). Global forces towards greater energy efficiency. Project Appraisal, 4(1), 9-16. doi: 10.1080/02688867.1989.9726700.

McKane, A., Therkelsen, P., Scodel, A., Prakash, R., Aghajanzadeh, A. y O'Sullivan, J. (2017). Predicting the quantifiable impacts of ISO 50001 on climate change mitigation, Energy Policy, 107, 278-288. doi: 10.1016/j. enpol.2017.04.049

Schudeleit, T, Züst, S., Weiss, L. y Wegener, K. (2016). The Total Energy Efficiency Index for machine tools, Energy, 102, 682-693. doi: 10.1016/j.energy.2016.02.126.

Scott, K., Roelich, K., Owen, A. y Barrett, J. (2017). Extending European energy efficiency standards to include material use: an analysis. Climate policy, 1-15, doi: 10.1080/14693062.2017.1333949.

Sundararaj, R., Karthick, A. y Ponrajkumar, R. (2016). Impact of ISO 50001-A study with reference to DCW Itd Sahupuram Plant in India. International Journal of Engineering Research and Management, 3(10), 112-115.

Therkelsena, P., Masanetb, E. y Worrellc, E. (2014). Energy efficiency opportunities in the U.S. commercial baking industry, Journal of Food Engineering, 130, 14-22. doi: 10.1016/j. jfoodeng.2014.01.004

Tallini,A. y Cedola, L. (2016). Evaluation Methodology for Energy Efficiency Measures in Industry and Service Sector, Energy Procedia, 101, 542549. doi: 10.1016/j.egypro.2016.11.069.

Vásquez, C., Carrillo, A., Tona, M., Galíndez, M., Macias, K. y Concetta, E. (2017). Sistema de gestión energética y ambiental de Productos Alimex CA. Suma de Negocios, 8(18), 115-121. doi: 10.1016/j.sumneg.2017.11.003

Visscher, H., Meijer, F., Majcen, D. y Itard, L. (2016). Improved governance for energy efficiency in housing. Building Research \& Information, 44(5-6), 552-561. doi: 10.1080/09613218.2016.1180808. 